\title{
Dealing depolarization of light in Mueller matrices with scalar metrics
}

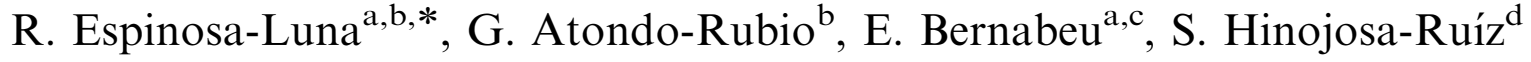 \\ ${ }^{a}$ Centro de Investigaciones en Óptica, A. C., Loma del Bosque 115, Colonia Lomas del Campestre, 37150 León, Guanajuato, \\ México \\ ${ }^{\mathrm{b}}$ Escuela de Ciencias Físico-Matemáticas, Universidad Autónoma de Sinaloa, Ciudad Universitaria s/n, 80010 Culiacán, Sinaloa, \\ México \\ ${ }^{\mathrm{c}}$ Departamento de Óptica, Universidad Complutense de Madrid, Facultad de Ciencias Físicas, Ciudad Universitaria s/n, \\ 28040 Madrid, Spain \\ ${ }^{\mathrm{d}}$ Facultad de Física, Universidad Autónoma de Zacatecas, Avenida Preparatoria 301, Fraccionamiento Progreso, 98060 Zacatecas, \\ Zacatecas, México
}

Received 20 October 2008; accepted 28 December 2008

\begin{abstract}
A number of depolarization metrics is applied to a series of reported Mueller matrices. It is shown the depolarization scalar metric $Q(M)$ provides consistent results with the reported scalar metrics like the depolarization index and the degree of polarization. It is shown $Q(M)$ provides additional information about the internal nature of the Mueller matrices, specifically when the upper limit, 3 , is reached. It is also shown the depolarization index and the $Q(M)$ metric are only necessary but not sufficient conditions for the physical realizability of Mueller matrices. Finally, $Q(M)$ is proven to be consistent in all cases studied here.
\end{abstract}

(C) 2009 Elsevier GmbH. All rights reserved.

Keywords: Optical physics; Polarization; Depolarization; Mueller matrices; Depolarization scalar metrics

\section{Introduction}

The concept of depolarization and the metrics to measure it have deserved a lot of interest over the last years [1-13]. In this work, the term depolarization refers to the loss in the degree of polarization after an incident polarized beam of light emerges from an optical system. The depolarization index $(0 \leqslant D I(M) \leqslant 1)$ has been defined as a single scalar metric associated to the Mueller matrix representing the depolarization of light

\footnotetext{
${ }^{*}$ Corresponding author at: Centro de Investigaciones en Óptica, A. C., Loma del Bosque 115, Colonia Lomas del Campestre, 37150 León, Guanajuato, México.

E-mail address: reluna@cio.mx (R. Espinosa-Luna).
}

associated to the linear response of an optical system [1-3]; it can be calculated directly from the Mueller matrix. The degree of polarization [10-13] is a measure of the percent of polarization associated to a beam of light $(0 \leqslant D o P \leqslant 1)$; it is measured directly from the beam of light under consideration, but it can be calculated also from a given Mueller matrix by considering, in addition, an incident Stokes vector. The degree of polarization and other derived metrics have been studied for a broad kind of systems and links with the diattenuation and the polarizance vectors have been analyzed [4]. The physical interpretation associated to the bounding limits for both metrics, $D I(M)$ and $\operatorname{DoP}(M, S)$, are the following: 0 corresponds to a totally depolarizing system, 1 to a non-depolarizing system, and 
intermediate values correspond to a partially depolarizing system. A recently reported metric, named $Q(M)$, has bounds associated which allows to identify a Mueller matrix as totally depolarizing, partially depolarizing, non-depolarizing diattenuating, and non-depolarizing non-diattenuating, respectively $[7,8]$.

A number of depolarization metrics is applied to a series of reported Mueller matrices. It is shown the depolarization scalar metric $Q(M)$ provides consistent results with the reported scalar metrics like the depolarization index and the degree of polarization. It is shown $Q(M)$ provides additional information about the internal nature of the Mueller matrices, specifically when the upper limit, 3 , is reached. It is also shown the depolarization index and the $Q(M)$ metric are only necessary but not sufficient conditions for the physical realizability of Mueller matrices. The overpolarization condition is the physical condition a Mueller matrix must fulfill in order to be physically consistent. Finally, $Q(M)$ is proven to be consistent in all cases studied here.

\section{Basic relations}

The linear response of an optical system to an incident Stokes vector can be expressed in terms of intensities, through the relation

$$
\begin{aligned}
S^{o} & =M S^{i} \Rightarrow\left(\begin{array}{c}
s_{0}^{o} \\
s_{1}^{o} \\
s_{2}^{o} \\
s_{3}^{o}
\end{array}\right)=\left[\begin{array}{llll}
m_{00} & m_{01} & m_{02} & m_{03} \\
m_{10} & m_{11} & m_{12} & m_{13} \\
m_{20} & m_{21} & m_{22} & m_{23} \\
m_{30} & m_{31} & m_{32} & m_{33}
\end{array}\right]\left(\begin{array}{c}
s_{0}^{i} \\
s_{1}^{i} \\
s_{2}^{i} \\
s_{3}^{i}
\end{array}\right) \\
& =\left(\begin{array}{l}
m_{00} s_{0}^{i}+m_{01} s_{1}^{i}+m_{02} s_{2}^{i}+m_{03} s_{3}^{i} \\
m_{10} s_{0}^{i}+m_{11} s_{1}^{i}+m_{12} s_{2}^{i}+m_{13} s_{3}^{i} \\
m_{20} s_{0}^{i}+m_{21} s_{1}^{i}+m_{22} s_{2}^{i}+m_{23} s_{3}^{i} \\
m_{30} s_{0}^{i}+m_{31} s_{1}^{i}+m_{32} s_{2}^{i}+m_{33} s_{3}^{i}
\end{array}\right),
\end{aligned}
$$

where $M$ is called the Mueller matrix of the system, represented as a $4 \times 4$ matrix of real elements, and $S$ is the Stokes vector. $S$ represents the polarization state of light, defined in terms of the orthogonal components of the electric field vector $\left(E_{p}, E_{s}\right)$ as

$$
S^{a}=\left(\begin{array}{c}
s_{0}^{a} \\
s_{1}^{a} \\
s_{2}^{a} \\
s_{3}^{a}
\end{array}\right)=\left(\begin{array}{c}
\left\langle E_{p}^{a} E_{p}^{a *}\right\rangle+\left\langle E_{s}^{a} E_{s}^{a *}\right\rangle \\
\left\langle E_{p}^{a} E_{p}^{a *}\right\rangle-\left\langle E_{s}^{a} E_{s}^{a *}\right\rangle \\
\left\langle E_{p}^{a} E_{s}^{a *}\right\rangle+\left\langle E_{s}^{a} E_{p}^{a *}\right\rangle \\
\pm i\left(\left\langle E_{p}^{a} E_{s}^{a *}\right\rangle-\left\langle E_{s}^{a} E_{p}^{a *}\right\rangle\right)
\end{array}\right),
$$

where $a=$ inc or scatt. Angular brackets represent temporal averages and * indicates complex conjugation, $i^{2}=-1$ is the complex number. The upper (lower) sign in the right hand side of $s_{3}^{a}$ corresponds to a description of polarization states as looking to the source (propagation direction). The normalized Stokes vectors can also be written in terms of the azimuthal $(0 \leqslant \psi \leqslant \pi)$ and the ellipticity $(-(\pi / 4) \leqslant \chi \leqslant(\pi / 4))$ angles of the polarization ellipse of the wave, respectively $[12,13]$

$S=\left\langle s_{0}\right\rangle\left(\begin{array}{c}1 \\ \cos (2 \chi) \cos (2 \psi) \\ \cos (2 \chi) \sin (2 \psi) \\ \sin (2 \chi)\end{array}\right)$

where $\left\langle s_{0}\right\rangle$ represents the intensity associated to the Stokes parameters; usually, it is fixed to the unity value. An interesting characteristic associated to an optical system is its capability to depolarize light, which is measured by using some of the following depolarization scalar metrics. The depolarization index $D I(M)$ and its physical realizable limits are defined by $[1,2]$ :

$0 \leqslant D I(M)=\left\{\sum_{j, k=0}^{3} m_{j k}^{2}-m_{00}^{2}\right\}^{1 / 2} / \sqrt{3} m_{00} \leqslant 1$.

$D I(M)$ is directly related to the Mueller matrix elements only. The degree of polarization, $\operatorname{DoP}(M, S)$, and its physical realizable limits have been defined by [10-13]

$0 \leqslant \operatorname{DoP}(M, S)$

$$
\begin{aligned}
& =\frac{\sqrt{\left(s_{1}^{o}\right)^{2}+\left(s_{2}^{o}\right)^{2}+\left(s_{3}^{o}\right)^{2}}}{s_{0}^{o}} \\
& =\frac{\left[\sum_{j=1}^{3}\left(m_{j 0} s_{0}^{i}+m_{j 1} s_{1}^{i}+m_{j 2} s_{2}^{i}+m_{j 3} s_{3}^{i}\right)^{2}\right]^{1 / 2}}{m_{00} s_{0}^{i}+m_{01} s_{1}^{i}+m_{02} s_{2}^{i}+m_{03} s_{3}^{i}} \leqslant 1 .
\end{aligned}
$$

$\operatorname{Do} P(M, S)$ is related with both, the Mueller matrix elements of the system under study and the incident Stokes vector. The diattenuation, $D(M)$, and the polarizance parameters, $P(M)$, are defined by [4]

$0 \leqslant D(M)=\sqrt{m_{01}^{2}+m_{02}^{2}+m_{03}^{2}} / m_{00} \leqslant 1$

and

$0 \leqslant P(M)=\sqrt{m_{10}^{2}+m_{20}^{2}+m_{30}^{2}} / m_{00} \leqslant 1$,

respectively.

The $Q(M)$ metric and its physical realizable bounds are defined as $[7,8]$

$$
\begin{aligned}
0 & \leqslant Q(M)=\frac{\sum_{j=1, k=0}^{3} m_{j k}^{2}}{\sum_{k=0}^{3} m_{0 k}^{2}}=\frac{3[D I(M)]^{2}-[D(M)]^{2}}{1+[D(M)]^{2}} \\
& =\frac{\left\{\sum_{j, k=1}^{3} m_{j k}^{2}\right\} / m_{00}^{2}+[P(M)]^{2}}{1+[D(M)]^{2}} \leqslant 3,
\end{aligned}
$$

where $Q(M)=0$ for a totally depolarizing optical system; $0<Q(M)<1$ for a partially depolarizing optical system; if $1 \leqslant Q(M)<3$ and $0<D I(M)<1$ the system 
partially depolarizes also, but if $D I(M)=1$, it is a nondepolarizing diattenuating optical system; and $Q(M)=$ 3 for a non-depolarizing non-diattenuating optical system, respectively [7,8].

A necessary and sufficient scalar condition for a Mueller matrix to be derivable from a Jones matrix has been reported to be given by the Gil-Bernabeu theorem $[1,2,9]$

$\operatorname{Tr}\left(M^{\mathrm{T}} M\right)=4 m_{00}^{2}$.

In a recently reported work, Gil has shown Eq. (8) is valid for any deterministic system [9]. We will take this result as valid in the rest of this work.

Following the development of Brosseau [13] and using the function,

$$
\begin{aligned}
F_{j}\left(\chi_{i n c}, \psi_{i n c}\right)= & m_{j 0}+m_{j 1} \cos \left(2 \chi_{i n c}\right) \cos \left(2 \psi_{i n c}\right) \\
& +m_{j 2} \cos \left(2 \chi_{i n c}\right) \sin \left(2 \psi_{i n c}\right)+m_{j 3} \sin \left(2 \chi_{i n c}\right)
\end{aligned}
$$

the overpolarization condition is given as [13]:

$$
\begin{aligned}
0 & \leqslant P_{0}\left(\chi_{i n c}, \psi_{i n c}\right)=\frac{1}{F_{0}\left(\chi_{i n c}, \psi_{i n c}\right)}\left(\sum_{j=1}^{3}\left(F_{j}\left(\chi_{i n c}, \psi_{i n c}\right)\right)^{2}\right)^{1 / 2} \\
& \leqslant 1 .
\end{aligned}
$$

For the case of a specific given Mueller matrix, a usual procedure is just to scan for all the possible incident Stokes vectors whose outputs can be associated to physically realizable Stokes vectors (overpolarization condition) [13]. That condition can be plotted in threedimensions as a function of the incident state of polarization parametrized by the angles $0 \leqslant \psi \leqslant \pi$ (azimuth) and $-(\pi / 4) \leqslant \chi \leqslant(\pi / 4)$ (ellipticity) of the polarization ellipse of the wave, respectively [13]. In practice, the experimentalists that use the ideal polarimeter arrangement usually deal with any of the six basic polarization states to determine the Mueller matrix of a given optical system $[14,15]$. The ideal polarimeter arrangement employs classical optical elements like linear polarizers and $\frac{1}{4}$-wave retardation plates for the generation and the analysis of the polarization states, respectively. That kind of experimental setup is handled manually. The six basic polarization states are the linear polarization parallel $(\mathrm{p})$, perpendicular $(\mathrm{s})$, to $+45^{\circ}(+)$ and to $-45^{\circ}(-)$ respect to the incidence plane, respectively, and the circular right-(r) and left-handed (l) polarization, respectively. The results obtained in this work are presented by using tables and graphics in the next Section. We have obtained the degree of polarization for each Mueller matrix by using each of the six basic polarization incident states considered here and its maximum value also, in addition to the single scalar polarization metrics $D I(M), P(M), D(M)$ and $Q(M)$.

\section{Results}

The Table 1 contains the results obtained by applying Eqs. (3) and (7) to the general Mueller matrices associated to ideal polarizers and ideal wave-retarders (see Appendix A [16]).

According to Eq. (3) all the matrices, except $M_{c}, M_{d}, M_{e}$, are non-depolarizing Mueller matrices, $M_{c}$ is a totally depolarizing matrix, and $M_{d}, M_{e}$ are partial depolarizing systems. On the other hand, the $Q(M)$ metric, Eq. (7), establishes that $M_{a}, M_{b}, M_{f}, M_{g}$ are non-depolarizing and non-diattenuating Mueller matrices; $M_{h}, M_{i}, M_{j}$ are non-depolarizing Mueller matrices and depending on the maximum and minimum values assigned to the transmittances $q$ and $r$, respectively, they can be more or less diattenuating. Observe that $Q(M)$ is sensible to the internal nature of the Mueller matrices associated to these optical systems. The results obtained in Table 1 are trivial; however, we have considered them as a way to verify the consistency of the $Q(M)$ metric and some advantage with respect to the depolarization index, Eq. (3).

Table 2 shows the results obtained from the application of Eqs. (3)-(7), the Gil-Bernabeu theorem, Eq. (8), and the overpolarization condition, Eq. (10), to different published Mueller matrices (see Appendix B). According to the $Q(M)$ depolarization scalar metric, all of these matrices have values inside $0<Q(M)<1$. This means they are associated to partially depolarizing optical systems. Note this interpretation is consistent with the results provided by expressions (3)-(6), (8), (10), presented in Table 2. The value of $D o P_{\operatorname{Max}}$ is the maximum value obtained when Eq. (10) is applied to all

Table 1. Single scalar metrics for matrices associated to ideal optical elements (polarizers and wave-retarders).

\begin{tabular}{lll}
\hline & $D I(M)$ & $Q(M)$ \\
\hline$M_{a}$ Non-polarizing element & 1 & 3 \\
$M_{b}$ Absorber & 1 & 3 \\
$M_{c}$ Ideal depolarizer & 0 & 0 \\
$M_{d}$ Non-uniform depolarizer & $\sqrt{\frac{a^{2}+b^{2}+c^{2}}{3}}$ & $a^{2}+b^{2}+c^{2}$ \\
$M_{e}$ Uniform depolarizer & $\alpha$ & $3 \alpha^{2}$ \\
$M_{f}$ Linear retarder, fast axis $\theta$, & 1 & 3 \\
retardance $\delta$ & & \\
$M_{g}$ Circular retarder, retardance $\delta$ & 1 & 3 \\
$M_{h}$ Linear diattenuator, axis $\theta$, & 1 & $1+\frac{4 q r}{q^{2}+r^{2}}$ \\
Int. transmittances $q, r$ & & $1+\frac{4 q r}{q^{2}+r^{2}}$ \\
$M_{i}$ Circular diattenuator, Int. & 1 & $1+\frac{4 q r}{q^{2}+r^{2}}$ \\
transmittances $q, r$ & & \\
$M_{j}$ Linear diattenuator and & 1 & \\
retarder, fast axis $0^{\circ}$, Int. & & \\
transmittances $(q, r)$, and & & \\
retardance $\delta$ & &
\end{tabular}

The Mueller matrices were taken from Appendix A [16]. 
Table 2. Single scalar metrics for the Mueller matrices considered in Appendix B.

\begin{tabular}{|c|c|c|c|c|c|c|c|c|c|c|}
\hline & $M 1$ & $M 2$ & M3 & $M 4$ & M5 & M6 & $M 7$ & $M 8$ & $M 9$ & $M 10$ \\
\hline$D_{o} P_{p}$ & 0.5119 & 0.5123 & 0.4976 & 0.2104 & 0.3250 & 0.748 & 0.740 & 0.273 & 0.269 & 0.9976 \\
\hline $\mathrm{DoP}_{s}$ & 0.7053 & 0.7060 & 0.6519 & 0.2041 & 0.3222 & 0.796 & 0.785 & 0.257 & 0.265 & 0.9950 \\
\hline $\mathrm{DoP}_{+45}$ & 0.4593 & 0.4585 & 0.4413 & 0.2224 & 0.3275 & 0.526 & 0.439 & 0.273 & 0.273 & 0.0100 \\
\hline$D o P_{-45}$ & 0.4028 & 0.4022 & 0.5256 & 0.2233 & 0.3283 & 0.426 & 0.552 & 0.209 & 0.227 & 0.0096 \\
\hline$D o P_{r}$ & 0.4416 & 0.4533 & 0.4223 & 0.1275 & 0.2734 & 0.416 & 0.336 & 0.603 & 0.605 & 0.0040 \\
\hline$D_{o} P_{l}$ & 0.5095 & 0.5243 & 0.4774 & 0.1349 & 0.2778 & 0.339 & 0.394 & 0.480 & 0.479 & 0.0069 \\
\hline$P(M)$ & 0.0145 & 0.0026 & 0.0851 & 0.0085 & 0.0105 & 0.121 & 0.074 & 0.047 & 0.069 & 0.0015 \\
\hline$D(M)$ & 0.1846 & 0.1903 & 0.0397 & 0.0231 & 0.0270 & 0.134 & 0.000 & 0.046 & 0.000 & 0.0022 \\
\hline$D I(M)$ & 0.5144 & 0.5152 & 0.5017 & 0.1917 & 0.3103 & 0.569 & 0.564 & 0.373 & 0.375 & 0.5753 \\
\hline$Q(M)$ & 0.7348 & 0.7334 & 0.7524 & 0.1097 & 0.2879 & 0.936 & 0.955 & 0.416 & 0.423 & 0.9928 \\
\hline$D_{o} P_{\operatorname{Max}}$ & 0.7050 & 0.7130 & 0.6592 & 0.2240 & 0.3340 & 0.828 & 0.793 & 0.612 & 0.605 & 1.0000 \\
\hline $\operatorname{Tr}\left(M^{\mathrm{T}} M\right) / 4 m_{00}^{2}$ & 0.448 & 0.449 & 0.438 & 0.2776 & 0.3222 & 0.493 & 0.488 & 0.354 & 0.355 & 0.4982 \\
\hline
\end{tabular}

Note that $0<Q(M)<1$ for all of them, which mean they depolarize light partially.

the incident Stokes vectors taken from the Poincaré sphere. In this case, all the matrices $M 1-M 10$ are physically consistent.

All the numbers reported in this work have been calculated by considering the approximation at which the Mueller matrices were originally reported.

In Table 3a are shown the results when Eqs. (3)-(8), (10) are applied to the Mueller matrices considered in Appendix C. Note that $1 \leqslant Q(M)<3$ and $0<D I(M)<1$ for all of them, which mean they depolarize light partially. In this case, $Q(M)=1$. This result is consistent with the remaining scalar metrics, Eqs. (3)-(6), the Gil-Bernabeu theorem, Eq. (8), and the overpolarization condition, Eq. (10). Note all of them are physically consistent Mueller matrices.

In Table $3 \mathrm{~b}$ are shown results when Eqs. (3)-(8), (10) are applied to the Mueller matrices considered in Appendix D. Note that $1 \leqslant Q(M)<3$ and $0<D I(M)<1$ for all of them, which mean they depolarize light partially. This result is consistent with the remaining scalar metrics.

Observe that $M 14-M 16, M 18, M 21, M 22$ have associated physically consistent values for the depolarization index, Eq. (3), the diattenuation and the polarizance parameters, Eqs. (5) and (6), the $Q(M)$ metric, Eq. (7), and the Gil-Bernabeu theorem, Eq. (8); however, there exist some incident Stokes vectors that generate an unphysical output degree of polarization $\left(D o P_{M a x}>1\right)$. The remaining matrices, $M 17, M 19, M 20$, fulfill all the physical conditions considered here.

When Eqs. (3)-(8) and (10) are applied to the Mueller matrices shown in the Appendix E, Table 4 is obtained. All of these matrices have values $1 \leqslant Q(M)<3$ and $D I(M)=1$. According to the metric $Q(M)$, they are associated to non-depolarizing diattenuating optical systems (note they have a non zero diattenuation parameter). According to the depolarization index, Eq. (3), all of them are associated to non-depolarizing systems, but this metric does not adds additional information about the internal nature of the matrices studied. Matrices $M 23, M 24, M 26, M 30-M 32$ and slightly $M 25$ are physically consistent, according to the overpolarization condition, Eq. (10). Matrices M27-M29 are not physically consistent, following Eq. (10). The Gil-Bernabeu theorem states that all the matrices, with exception to $M 25, M 27, M 28$, are associated to non-depolarizing optical systems and can be derived from a Jones matrix (they are Mueller-Jones matrices). Observe that the depolarization index, the $Q(M)$ metric and the Gil-Bernabeu theorem are only necessary but no sufficient conditions for the physical realizability of Mueller matrices.

Table 5 shows values of the single scalar metrics for the Mueller matrices considered in Appendix F. Note that $Q(M) \approx 3$ for all of them, which mean they are associated to non-depolarizing non-diattenuating systems. This conclusion is consistent with the depolarization index, Eq. (3), the diattenuation and the polarizance parameters, Eqs. (5) and (6), and they are physically realizable, according to the overpolarization condition, Eq. (10). Finally, all of them are Mueller-Jones matrices associated to non-diattenuating Jones matrices, following the Gil-Bernabeu theorem, Eq. (8). In other words, matrices M33-M37 are pure Mueller matrices [9].

Table 6 shows the Mueller matrices with $Q(M)>3$ (see Appendix G). According to this metric, matrices M38-M41 are not physically consistent. The same conclusion is confirmed by using the depolarization index and the degree of polarization. Note that these Mueller matrices have physical diattenuation and polarizance parameters within their validity range. This means these parameters are only necessary but not sufficient conditions for the physical consistency of Mueller matrices. 
Table 3. (a) Mueller matrices with $1 \leqslant Q(M)<3$ and $0<D I(M)<1, Q(M)=1$. (b) Mueller matrices with $1 \leqslant Q(M)<3$ and $0<D I(M)<1$.

\begin{tabular}{|c|c|c|c|c|c|c|c|c|c|}
\hline & & & $M 11$ & & & $M 12$ & & & $M 13$ \\
\hline \multicolumn{10}{|l|}{ (a) } \\
\hline$D_{o} P_{p}$ & & & 1.00 & & & 1.00 & & & 1.0000 \\
\hline$D_{o} P_{s}$ & & & 1.00 & & & 1.00 & & & 1.0000 \\
\hline$D_{o} P_{+45}$ & & & 0.03 & & & 0.06 & & & 0.0956 \\
\hline$D_{o} P_{-45}$ & & & 0.03 & & & 0.06 & & & 0.0956 \\
\hline$D o P_{r}$ & & & 0.03 & & & 0.06 & & & 0.0956 \\
\hline$D_{o} P_{l}$ & & & 0.03 & & & 0.06 & & & 0.0956 \\
\hline$P(M)$ & & & 0.03 & & & 0.06 & & & 0.0956 \\
\hline$D(M)$ & & & 0.03 & & & 0.06 & & & 0.0956 \\
\hline$D I(M)$ & & & 0.57 & & & 0.57 & & & 0.5826 \\
\hline$Q(M)$ & & & 1.00 & & & 1.00 & & & 1.0000 \\
\hline$D o P_{\operatorname{Max}}$ & & & 1.00 & & & 1.00 & & & 1.0000 \\
\hline \multirow[t]{2}{*}{$\operatorname{Tr}\left(M^{\mathrm{T}} M\right) / 4 m_{00}^{2}$} & & & 0.50 & & & 0.50 & & & 0.5046 \\
\hline & $M 14$ & $M 15$ & $M 16$ & $M 17$ & $M 18$ & M19 & $M 20$ & $M 21$ & $M 22$ \\
\hline \multicolumn{10}{|l|}{ (b) } \\
\hline$D_{o} P_{p}$ & 0.7252 & 0.6339 & 1.0130 & 0.7312 & 0.955 & 0.8044 & 0.7501 & 0.974 & 0.985 \\
\hline $\mathrm{DoP}_{s}$ & 0.7738 & 0.8039 & 0.9801 & 0.7104 & 1.002 & 0.7307 & 0.6891 & 0.968 & 0.968 \\
\hline$D o P_{+45}$ & 0.8405 & 0.8044 & 0.9583 & 0.7027 & 0.957 & 0.9386 & 0.8819 & 0.945 & 0.961 \\
\hline$D o P_{-45}$ & 0.8308 & 0.7307 & 1.0109 & 0.7611 & 0.980 & 0.8609 & 0.8043 & 1.046 & 1.008 \\
\hline$D o P_{r}$ & 0.9320 & 0.9386 & 1.0055 & 0.6581 & 1.006 & 0.7892 & 0.7537 & 0.934 & 0.993 \\
\hline$D_{o} P_{l}$ & 0.9197 & 0.8609 & 0.9870 & 0.5737 & 0.998 & 0.6979 & 0.6718 & 0.941 & 0.955 \\
\hline$P(M)$ & 0.0885 & 0.1867 & 0.0103 & 0.0576 & 0.007 & 0.1867 & 0.1816 & 0.048 & 0.026 \\
\hline$D(M)$ & 0.0901 & 0.1804 & 0.0303 & 0.0583 & 0.032 & 0.1804 & 0.1791 & 0.064 & 0.000 \\
\hline$D I(M)$ & 0.8426 & 0.8080 & 0.9923 & 0.6913 & 0.983 & 0.8080 & 0.7703 & 0.968 & 0.968 \\
\hline$Q(M)$ & 2.1049 & 1.8654 & 2.9505 & 1.4254 & 2.896 & 1.8654 & 1.6939 & 2.796 & 2.814 \\
\hline$D_{o} P_{M a x}$ & 1.1278 & 1.1035 & 1.0409 & 0.7728 & 1.012 & 0.9882 & 0.9030 & 1.084 & 1.072 \\
\hline $\operatorname{Tr}\left(M^{\mathrm{T}} M\right) / 4 m_{00}^{2}$ & 0.7825 & 0.7397 & 0.9885 & 0.6084 & 0.975 & 0.7397 & 0.6951 & 0.952 & 0.953 \\
\hline
\end{tabular}

Partially depolarizing optical systems.

Table 4. Mueller matrices with $1 \leqslant Q(M)<3$ and $D I(M)=1$.

\begin{tabular}{|c|c|c|c|c|c|c|c|c|c|c|}
\hline & $M 23$ & $M 24$ & $M 25$ & $M 26$ & $M 27$ & $M 28$ & $M 29$ & $M 30$ & M31 & M32 \\
\hline$D_{o} P_{p}$ & 0.999 & 1.00 & 1.0003 & 1.00 & 1.000 & 0.999 & 1.000 & 1.000 & 1.000 & 0.999 \\
\hline$D_{o} P_{s}$ & 1.000 & 1.00 & 1.0003 & 1.00 & 1.000 & 0.999 & 1.000 & 1.000 & 1.000 & 0.999 \\
\hline$D o P_{+45}$ & 1.000 & 1.00 & 1.0003 & 1.00 & 1.000 & 0.997 & 0.999 & 0.999 & 1.001 & 1.000 \\
\hline$D_{o} P_{-45}$ & 1.000 & 1.00 & 1.0003 & 1.00 & 1.000 & 1.003 & 0.999 & 0.999 & 1.001 & 1.000 \\
\hline$D o P_{r}$ & 0.877 & 1.00 & 1.0000 & 1.00 & 0.999 & 1.000 & 0.999 & 1.000 & 1.000 & 1.000 \\
\hline$D_{o} P_{l}$ & 0.929 & 1.00 & 1.0000 & 1.00 & 0.999 & 1.000 & 0.999 & 1.000 & 1.000 & 1.000 \\
\hline$P(M)$ & 0.067 & 0.28 & 0.2801 & 0.10 & 0.238 & 0.238 & 0.134 & 0.046 & 0.067 & 0.064 \\
\hline$D(M)$ & 0.067 & 0.28 & 0.2800 & 0.10 & 0.238 & 0.238 & 0.134 & 0.046 & 0.067 & 0.064 \\
\hline$D I(M)$ & 1.000 & 1.00 & 1.0002 & 1.00 & 1.001 & 1.001 & 1.000 & 1.000 & 1.000 & 1.000 \\
\hline$Q(M)$ & 2.981 & 2.70 & 2.7101 & 2.97 & 2.794 & 2.794 & 2.929 & 2.991 & 2.986 & 2.983 \\
\hline$D_{o} P_{M a x}$ & 1.000 & 1.00 & 1.0003 & 1.00 & 1.008 & 1.417 & 1.144 & 1.000 & 1.001 & 1.000 \\
\hline $\operatorname{Tr}\left(M^{\mathrm{T}} M\right) / 4 m_{00}^{2}$ & 1.000 & 1.00 & 1.0003 & 1.00 & 1.002 & 1.002 & 1.000 & 1.000 & 1.001 & 1.000 \\
\hline
\end{tabular}

Non-depolarizing diattenuating optical systems.

Figs. 1-4 show the graphics of the overpolarization condition, Eq. (10), for the matrices considered in Table 6. Note that there exist several incident Stokes vectors that originate a non-physically consistent output for the Mueller matrices $M 38-M 41\left(D_{o} P_{\operatorname{Max}}>1\right)$.

\section{Conclusions}

Several depolarization metrics has been applied to a large series of reported Mueller matrices in order to show that the depolarization scalar metric $Q(M)$ 
Table 5. Mueller matrices with $Q(M) \approx 3$.

\begin{tabular}{llllll}
\hline & $M 33$ & $M 34$ & $M 35$ & $M 36$ & $M 37$ \\
\hline$D o P_{p}$ & 1.000 & 1.000 & 1.000 & 0.999 & 0.999 \\
$D o P_{s}$ & 1.000 & 1.000 & 1.000 & 0.999 & 0.999 \\
$D o P_{+45}$ & 0.999 & 0.999 & 0.999 & 1.000 & 1.000 \\
$D o P_{-45}$ & 0.999 & 0.999 & 0.999 & 1.000 & 1.000 \\
$D o P_{r}$ & 0.999 & 1.000 & 1.000 & 0.999 & 0.999 \\
$D o P_{l}$ & 0.999 & 1.000 & 1.000 & 0.999 & 0.999 \\
$P(M)$ & 0.000 & 0.000 & 0.000 & 0.000 & 0.000 \\
$D(M)$ & 0.000 & 0.000 & 0.000 & 0.000 & 0.000 \\
$D I(M)$ & 0.999 & 1.000 & 1.000 & 0.999 & 1.000 \\
$Q(M)$ & 2.999 & 3.000 & 3.000 & 2.999 & 2.999 \\
$D o P_{M a x}$ & 1.000 & 1.000 & 1.000 & 1.000 & 1.000 \\
$\operatorname{Tr}\left(M^{\mathrm{T}} M\right) / 4 m_{00}^{2}$ & 0.999 & 1.000 & 1.000 & 0.999 & 1.000 \\
\hline
\end{tabular}

Non-depolarizing non-diattenuating optical systems.

Table 6. Mueller matrices with $Q(M)>3$.

\begin{tabular}{lllll}
\hline & $M 38$ & $M 39$ & $M 40$ & $M 41$ \\
\hline$D o P_{p}$ & 1.027 & 1.5326 & 1.022 & 0.979 \\
$D o P_{s}$ & 1.026 & 1.3980 & 1.022 & 0.999 \\
$D o P_{+45}$ & 1.022 & 0.9796 & 1.018 & 1.000 \\
$D o P_{-45}$ & 1.038 & 0.9923 & 1.024 & 1.201 \\
$D o P_{r}$ & 1.167 & 0.9540 & 1.016 & 0.999 \\
$D o P_{l}$ & 0.919 & 0.9335 & 1.016 & 0.994 \\
$P(M)$ & 0.026 & 0.1000 & 0.005 & 0.046 \\
$D(M)$ & 0.133 & 0.1000 & 0.006 & 0.057 \\
$D I(M)$ & 1.031 & 1.1542 & 1.020 & 1.028 \\
$Q(M)$ & 3.115 & 3.9472 & 3.122 & 3.158 \\
$D o P_{M a x}$ & 1.173 & 1.7524 & 1.027 & 1.212 \\
$\operatorname{Tr}\left(M^{\mathrm{T}} M\right) / 4 m_{00}^{2}$ & 1.047 & 1.2492 & 1.030 & 1.042 \\
\hline
\end{tabular}

Non-physically consistent Mueller matrices.

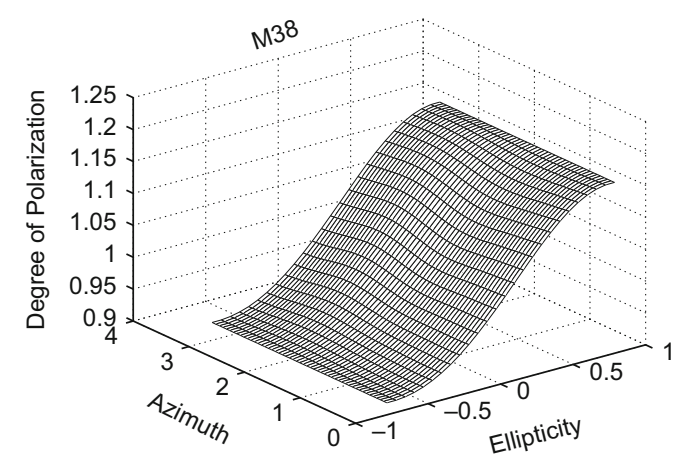

Fig. 1. Overpolarization condition, Eq. (10), for $M 38$ of Table 6 (see Appendix G). Non physically consistent optical system $\left(\right.$ Do $\left.P_{\text {Max }}>1\right)$.

provides consistent results with the reported scalar metrics like the depolarization index and the degree of polarization. At more $Q(M)$ provides additional information about the internal nature of the Mueller matrices, specifically when the upper limit, 3 , is reached. It has been shown also, the depolarization index, the

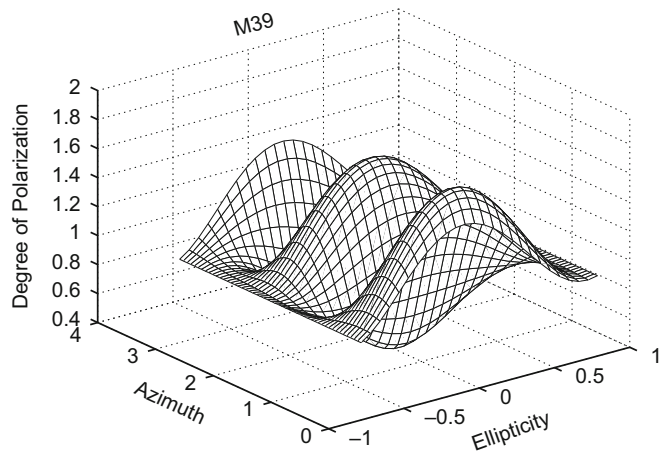

Fig. 2. Overpolarization condition, Eq. (10), for M39 of Table 6 (see Appendix G). Non physical consistent optical system $\left(\right.$ DoP $\left._{\text {Max }}>1\right)$.

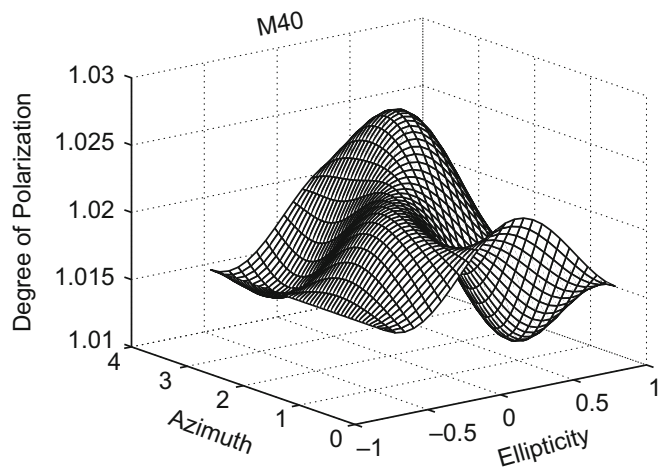

Fig. 3. Overpolarization condition, Eq. (10), for $M 40$ of Table 6 (see Appendix G). Non physical consistent optical system $\left(\right.$ Do $\left._{\text {Max }}>1\right)$.

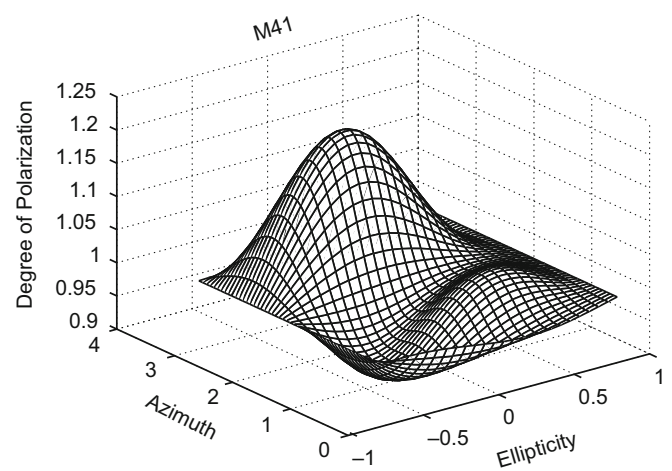

Fig. 4. Overpolarization condition, Eq. (10), for M41 of Table 6 (see Appendix G). Non physical consistent optical system $\left(\right.$ Do $\left._{\text {Max }}>1\right)$.

$Q(M)$ metric and the Gil-Bernabeu theorem are only necessary but not sufficient conditions for the physical realizability of Mueller matrices. The overpolarization condition is the physical condition a Mueller matrix must fulfill in order to be physically consistent. Finally, in all the cases studied here, $Q(M)$ has proven to be consistent. 


\section{Acknowledgments}

R. Espinosa-Luna and E. Bernabeu express their gratitude to CONACYT for the support provided for the realization of this work under project No. 83182. E. Bernabeu expresses his particular acknowledgments to
"Flores Valles" Program of UCM to facilitate his presence in CIO. R. Espinosa-Luna, G. Atondo-Rubio and S. Hinojosa-Ruíz express their gratitude to their respective Institutions for the realization of this work as part of the GIPYS initiative (Grupo Interinstitucional de Polarización y Scattering).

\section{Appendix A. Form of the Mueller matrices as taken from [16]:}

$$
\begin{aligned}
& M_{a}=\left(\begin{array}{cccc}
1 & 0 & 0 & 0 \\
0 & 1 & 0 & 0 \\
0 & 0 & 1 & 0 \\
0 & 0 & 0 & 1
\end{array}\right) ; \quad M_{b}=\left(\begin{array}{cccc}
\alpha & 0 & 0 & 0 \\
0 & \alpha & 0 & 0 \\
0 & 0 & \alpha & 0 \\
0 & 0 & 0 & \alpha
\end{array}\right) ; \\
& M_{c}=\left(\begin{array}{cccc}
1 & 0 & 0 & 0 \\
0 & 0 & 0 & 0 \\
0 & 0 & 0 & 0 \\
0 & 0 & 0 & 0
\end{array}\right) ; \quad M_{d}=\left(\begin{array}{cccc}
1 & 0 & 0 & 0 \\
0 & a & 0 & 0 \\
0 & 0 & b & 0 \\
0 & 0 & 0 & c
\end{array}\right) \\
& M_{e}=\left(\begin{array}{cccc}
1 & 0 & 0 & 0 \\
0 & \alpha & 0 & 0 \\
0 & 0 & \alpha & 0 \\
0 & 0 & 0 & \alpha
\end{array}\right) ; \quad M_{g}=\frac{1}{2}\left[\begin{array}{cccc}
1 & 0 & 0 & 0 \\
0 & \cos \delta & \sin \delta & 0 \\
0 & -\sin \delta & \cos \delta & 0 \\
0 & 0 & 0 & 1
\end{array}\right] \text {; } \\
& M_{i}=\frac{1}{2}\left[\begin{array}{cccc}
q+r & 0 & 0 & q-r \\
0 & 2 \sqrt{q r} & 0 & 0 \\
0 & 0 & 2 \sqrt{q r} & 0 \\
q-r & 0 & 0 & q+r
\end{array}\right] \\
& M_{f}=\frac{1}{2}\left[\begin{array}{cccc}
1 & 0 & 0 & 0 \\
0 & \cos ^{2} 2 \theta+\sin ^{2} 2 \theta \cos \delta & \sin 2 \theta \cos 2 \theta(1-\cos \delta) & -\sin 2 \theta \sin \delta \\
0 & \sin 2 \theta \cos 2 \theta(1-\cos \delta) & \sin ^{2} 2 \theta+\cos ^{2} 2 \theta \cos \delta & \cos 2 \theta \sin \delta \\
0 & \sin 2 \theta \sin \delta & -\cos 2 \theta \sin \delta & \cos \delta
\end{array}\right] \\
& M_{h}=\frac{1}{2}\left[\begin{array}{cccc}
q+r & (q-r) \cos 2 \theta & (q-r) \sin 2 \theta & 0 \\
(q-r) \cos 2 \theta & (q+r) \cos ^{2} 2 \theta+2 \sqrt{q r} \sin ^{2} 2 \theta & (q+r-2 \sqrt{q r}) \sin 2 \theta \cos 2 \theta & 0 \\
(q-r) \sin 2 \theta & (q+r-2 \sqrt{q r}) \sin 2 \theta \cos 2 \theta & (q+r) \sin ^{2} 2 \theta+2 \sqrt{q r} \cos ^{2} 2 \theta & 0 \\
0 & 0 & 0 & 2 \sqrt{q r}
\end{array}\right] \\
& M_{j}=\frac{1}{2}\left[\begin{array}{cccc}
q+r & q-r & 0 & 0 \\
q-r & q+r & 0 & 0 \\
0 & 0 & 2 \sqrt{q r} \cos \delta & 2 \sqrt{q r} \sin \delta \\
0 & 0 & -2 \sqrt{q r} \sin \delta & 2 \sqrt{q r} \cos \delta
\end{array}\right]
\end{aligned}
$$


Appendix B. Mueller matrices employed to calculate Table 2. Matrices $M 1, M 2$ were taken from [17], M3 from [18], M4, M5 from [19], $M 6-M 9$ from [20], and M10 from [21]

$$
\begin{aligned}
& M 1=\left[\begin{array}{cccc}
1.0000 & 0.1631 & -0.0322 & 0.0802 \\
0.0083 & 0.4038 & 0.2555 & -0.2158 \\
-0.0026 & 0.4297 & -0.1376 & 0.2016 \\
-0.0116 & 0.0597 & -0.3175 & -0.3690
\end{array}\right], \\
& M 2=\left[\begin{array}{cccc}
1.0000 & 0.1633 & -0.0655 & 0.0725 \\
0.0018 & 0.4042 & 0.2324 & -0.2324 \\
0.0019 & 0.4302 & -0.1745 & 0.2624 \\
0.0003 & 0.0598 & -0.3149 & -0.3170
\end{array}\right] \text {, } \\
& M 3=\left[\begin{array}{cccc}
1 & 0.0262 & 0.0169 & 0.0246 \\
-0.0711 & 0.5573 & -0.0001 & -0.0789 \\
-0.0389 & -0.1171 & 0.4708 & 0.0457 \\
-0.0260 & 0.0185 & -0.0728 & 0.4318
\end{array}\right] \text {, } \\
& M 4=\left[\begin{array}{cccc}
1.0000 & 0.0227 & -0.0031 & -0.0028 \\
0.0077 & 0.2066 & -0.0038 & -0.0096 \\
0.0009 & -0.0121 & -0.2225 & -0.0024 \\
0.0035 & 0.0118 & -0.0082 & -0.1306
\end{array}\right] \text {, } \\
& M 5=\left[\begin{array}{cccc}
1.0000 & 0.0269 & -0.0021 & -0.0018 \\
0.0101 & 0.3236 & -0.0087 & -0.0023 \\
0.0008 & -0.0024 & -0.3276 & 0.0009 \\
0.0026 & 0.0023 & -0.0029 & -0.2754
\end{array}\right] \text {, } \\
& M 6=\left[\begin{array}{cccc}
1 & -0.115 & -0.066 & 0.023 \\
-0.111 & 0.759 & -0.061 & -0.001 \\
-0.018 & 0.151 & -0.435 & -0.139 \\
-0.046 & 0.006 & 0.128 & -0.334
\end{array}\right] \text {, } \\
& M 7=\left[\begin{array}{cccc}
1 & 0 & 0 & 0 \\
-0.028 & 0.756 & -0.072 & 0.021 \\
-0.062 & -0.072 & 0.488 & -0.014 \\
-0.03 & 0.021 & -0.014 & 0.358
\end{array}\right] \text {, } \\
& M 8=\left[\begin{array}{cccc}
1 & -0.009 & -0.021 & -0.041 \\
-0.002 & 0.256 & -0.029 & -0.003 \\
0.024 & 0.045 & 0.235 & -0.032 \\
0.041 & 0.024 & 0.017 & 0.538
\end{array}\right] \text {, }
\end{aligned}
$$

$$
\begin{aligned}
M 9= & {\left[\begin{array}{cccc}
1 & 0 & 0 & 0 \\
-0.001 & 0.258 & 0.01 & 0.009 \\
0.028 & 0.01 & 0.241 & -0.015 \\
0.064 & 0.009 & -0.015 & 0.541
\end{array}\right], } \\
M 10= & {\left[\begin{array}{cccc}
1 & -0.0013 & -0.0015 & -0.0010 \\
0.00 & 0.9963 & -0.0083 & -0.0005 \\
-0.0007 & 0.0068 & -0.0049 & 0.0029 \\
0.0013 & 0.0033 & -0.0013 & -0.0046
\end{array}\right] . }
\end{aligned}
$$

Appendix C. Mueller matrices used to calculate Table 3a. Matrices M11 - M13 were taken from [22]

$$
\begin{aligned}
& M 11=\left[\begin{array}{cccc}
0.90 & 0.03 & 0 & 0 \\
0.03 & 0.90 & 0 & 0 \\
0 & 0 & 0 & 0 \\
0 & 0 & 0 & 0
\end{array}\right], \\
& M 12=\left[\begin{array}{cccc}
0.80 & 0.05 & 0 & 0 \\
0.05 & 0.80 & 0 & 0 \\
0 & 0 & 0 & 0 \\
0 & 0 & 0 & 0
\end{array}\right], \\
& M 13=\left[\begin{array}{ccccc}
0.7215 & 0.069 & 0 & 0 \\
0.069 & 0.7215 & 0 & 0 \\
0 & 0 & 0 & 0 \\
0 & 0 & 0 & 0
\end{array}\right] .
\end{aligned}
$$

Appendix D. Mueller matrices employed to calculate Table 3b. Matrices M14, M15 were taken from [22], M16, M17 from [18], M18 from page 576 of [12], $M 19, M 20$ from [23], and M21, M22 from [20]

$$
\begin{aligned}
M 14 & =\left[\begin{array}{cccc}
0.8886 & -0.0131 & 0.0055 & 0.0786 \\
-0.0115 & 0.5762 & -0.2820 & -0.1668 \\
0.0048 & -0.2809 & 0.6825 & 0.0026 \\
0.0775 & -0.1672 & 0.0012 & 0.8061
\end{array}\right], \\
M 15 & =\left[\begin{array}{cccc}
0.7599 & -0.0623 & 0.0295 & 0.1185 \\
-0.0573 & 0.4687 & -0.1811 & -0.1863 \\
0.0384 & -0.1714 & 0.5394 & 0.0282 \\
0.1240 & -0.2168 & -0.0120 & 0.6608
\end{array}\right],
\end{aligned}
$$




$$
\begin{aligned}
& M 16=\left[\begin{array}{cccc}
1 & -0.0118 & 0.0279 & 0.0001 \\
0.0045 & 0.9956 & 0.0013 & 0.0350 \\
0.0012 & 0.0341 & 0.9838 & 0.0083 \\
0.0092 & 0.0178 & -0.0002 & 0.9956
\end{array}\right], \\
& M 17=\left[\begin{array}{cccc}
1 & -0.0146 & 0.0509 & 0.0243 \\
0.0004 & 0.7163 & 0.0268 & -0.0250 \\
0.0078 & -0.0544 & 0.7277 & 0.0104 \\
0.0571 & 0.0010 & 0.0035 & 0.6163
\end{array}\right], \\
& M 18=\left[\begin{array}{cccc}
0.998 & 0.026 & 0.019 & -0.002 \\
0.002 & 0.976 & -0.030 & 0.009 \\
0.007 & 0.033 & 0.966 & -0.002 \\
0.002 & -0.004 & -0.002 & 1.0
\end{array}\right] \text {, } \\
& M 19=\left[\begin{array}{cccc}
0.7599 & 0.0295 & 0.1185 & -0.0623 \\
0.0384 & 0.5394 & 0.0282 & -0.1714 \\
0.1240 & -0.012 & 0.6608 & 0.2168 \\
-0.0573 & -0.1811 & -0.1863 & 0.4687
\end{array}\right] \text {, } \\
& M 20=\left[\begin{array}{cccc}
0.7599 & 0.0257 & 0.1206 & -0.0576 \\
0.0372 & 0.5285 & 0.0001 & -0.0496 \\
0.1208 & -0.0001 & 0.6184 & 0.1920 \\
-0.0554 & -0.0572 & -0.1794 & 0.4822
\end{array}\right] \text {, } \\
& M 21=\left[\begin{array}{cccc}
1.000 & 0.026 & 0.044 & -0.039 \\
0.029 & 0.962 & -0.144 & -0.047 \\
0.002 & 0.126 & 0.975 & 0.026 \\
-0.039 & 0.019 & 0.115 & 0.936
\end{array}\right] \text {, } \\
& M 22=\left[\begin{array}{cccc}
1 & 0 & 0 & 0 \\
0.008 & 0.976 & -0.01 & -0.021 \\
-0.023 & -0.01 & 0.982 & 0.073 \\
-0.009 & -0.022 & 0.073 & 0.941
\end{array}\right] \text {. }
\end{aligned}
$$

Appendix E. Mueller matrices employed to calculate Table 4. Matrices M23, M27-M32 were taken from [20], M24, M25 were taken from [12], pages 171 and 172, respectively, and M26 from [19]

$$
M 23=\left[\begin{array}{cccc}
1 & -0.06 & -0.031 & 0.0 \\
-0.06 & 0.856 & 0.284 & -0.43 \\
-0.031 & 0.266 & 0.475 & 0.837 \\
-0.001 & 0.442 & -0.831 & 0.331
\end{array}\right]
$$

$$
\begin{aligned}
M 24 & =\left[\begin{array}{cccc}
0.50 & 0.14 & 0 & 0 \\
0.14 & 0.50 & 0 & 0 \\
0 & 0 & 0.48 & 0 \\
0 & 0 & 0 & 0.48
\end{array}\right], \\
M 25 & =\left[\begin{array}{llll}
0.500000 & 0.024311 & 0.137873 & 0.000000 \\
0.024360 & 0.480725 & 0.003578 & 0.000000 \\
0.137900 & 0.003270 & 0.499521 & 0.000000 \\
0.000000 & 0.000000 & 0.000000 & 0.480000
\end{array}\right],
\end{aligned}
$$$$
M 26=\left[\begin{array}{cccc}
0.19 & 0.02 & 0 & 0 \\
0.02 & 0.19 & 0 & 0 \\
0 & 0 & -0.19 & 0 \\
0 & 0 & 0 & -0.19
\end{array}\right],
$$$$
\begin{aligned}
M 27 & =\left[\begin{array}{cccc}
1 & 0.09 & -0.093 & -0.2 \\
0.155 & 0.874 & 0.119 & -0.435 \\
-0.179 & 0.303 & 0.487 & 0.804 \\
0.029 & 0.310 & -0.837 & 0.383
\end{array}\right], \\
M 28 & =\left[\begin{array}{cccc}
1 & 0.09 & -0.09 & -0.2 \\
0.09 & 0.975 & -0.004 & -0.009 \\
-0.093 & -0.004 & 0.976 & 0.009 \\
-0.2 & -0.009 & 0.009 & 0.992
\end{array}\right],
\end{aligned}
$$$$
M 29=\left[\begin{array}{cccc}
1 & -0.115 & -0.066 & 0.023 \\
-0.115 & 0.998 & 0.004 & -0.001 \\
0.066 & 0.004 & 0.993 & -0.001 \\
0.023 & -0.001 & -0.001 & 0.991
\end{array}\right] \text {, }
$$$$
M 30=\left[\begin{array}{cccc}
1 & -0.009 & -0.021 & -0.041 \\
-0.009 & 0.999 & 0 & 0 \\
-0.021 & 0 & 0.999 & 0 \\
-0.041 & 0 & 0 & 1
\end{array}\right] \text {, }
$$$$
M 31=\left[\begin{array}{cccc}
1 & -0.06 & -0.031 & 0 \\
-0.06 & 1 & 0.001 & 0 \\
-0.031 & 0.001 & 1 & 0 \\
0 & 0 & 0 & 0.998
\end{array}\right] \text {, }
$$

$$
M 32=\left[\begin{array}{cccc}
1 & 0.026 & 0.044 & -0.039 \\
0.026 & 0.998 & 0.001 & -0.001 \\
0.044 & 0.001 & 0.999 & -0.001 \\
-0.039 & -0.001 & -0.001 & 0.999
\end{array}\right] \text {. }
$$


Appendix F. Mueller matrices used to calculate Table 5. Matrices $M 33-M 37$ were taken from [20]

$$
\begin{aligned}
M 33 & =\left[\begin{array}{cccc}
1 & 0 & 0 & 0 \\
0 & 0.985 & -0.184 & 0 \\
0 & -0.175 & -0.924 & -0.312 \\
0 & 0.057 & 0.306 & -0.95
\end{array}\right], \\
M 34 & =\left[\begin{array}{cccc}
1 & 0 & 0 & 0 \\
0 & 0.988 & -0.152 & -0.022 \\
0 & 0.151 & 0.986 & -0.067 \\
0 & 0.032 & 0.063 & 0.998
\end{array}\right], \\
M 35 & =\left[\begin{array}{cccc}
1 & 0 & 0 & 0 \\
0 & 0.857 & 0.283 & -0.431 \\
0 & 0.265 & 0.475 & 0.839 \\
0 & 0.443 & -0.833 & 0.332
\end{array}\right], \\
M 36 & =\left[\begin{array}{cccc}
1 & 0 & 0 & 0 \\
0 & 0.892 & 0.130 & -0.432 \\
0 & 0.32 & 0.492 & 0.809 \\
0 & 0.318 & -0.861 & 0.398
\end{array}\right], \\
M 37 & =\left[\begin{array}{cccc}
1 & 0 & 0 & 0 \\
0 & 0.99 & -0.138 & -0.027 \\
0 & 0.136 & 0.99 & -0.048 \\
0 & 0.033 & 0.044 & 0.998
\end{array}\right] .
\end{aligned}
$$

Appendix G. Mueller matrices employed to calculate Table 6. Matrices $M 38, M 40$ were taken from [12], page 175 and 174, respectively, $M 39$ from [22], and M41 from [24]

$$
\begin{aligned}
M 38 & =\left[\begin{array}{cccc}
1 & 0.019 & 0.021 & -0.130 \\
-0.024 & -0.731 & -0.726 & 0.005 \\
0.008 & 0.673 & -0.688 & -0.351 \\
-0.009 & 0.259 & -0.247 & 0.965
\end{array}\right], \\
M 39 & =\left[\begin{array}{cccc}
0.8488 & -0.0503 & 0.0294 & 0.0617 \\
-0.0503 & 0.8304 & 0.0913 & -0.0920 \\
0.0294 & 0.913 & 0.8277 & 0 \\
0.0617 & -0.0920 & 0 & 0.7947
\end{array}\right],
\end{aligned}
$$

$$
\begin{aligned}
M 40 & =\left[\begin{array}{cccc}
0.978 & 0 & 0.003 & 0.005 \\
0 & 1 & -0.007 & 0.006 \\
0 & 0.007 & 0.999 & -0.007 \\
0.005 & -0.003 & -0.002 & 0.994
\end{array}\right], \\
M 41 & =\left[\begin{array}{cccc}
0.946 & 0.019 & 0.048 & -0.016 \\
-0.024 & -0.848 & 0.322 & 0.314 \\
0.003 & -0.261 & 0.087 & -0.885 \\
0.037 & -0.293 & -0.981 & -0.071
\end{array}\right] .
\end{aligned}
$$

\section{References}

[1] J.J. Gil, E. Bernabeu, A depolarization criterion in Mueller matrices, Opt. Acta 32 (1985) 259-261.

[2] J.J. Gil, E. Bernabeu, Depolarization and polarization indexes of an optical system, Opt. Acta 33 (1986) 185-189.

[3] J.J. Gil, E. Bernabeu, Obtainment of the polarizing and retardation parameters of a nondepolarizing optical system from the polar decomposition of its Mueller matrices, Optik 76 (1987) 67-71.

[4] S.Y. Lu, R.A. Chipman, Mueller matrices and the degree of polarization, Opt. Commun. 146 (1998) 11-14.

[5] B. DeBoo, J. Sasian, R. Chipman, Degree of polarization surfaces and maps for analysis of depolarization, Opt. Express 12 (2004) 4941-4958.

[6] R. Chipman, Depolarization index and the average degree of polarization, Appl. Opt. 44 (2005) 2490-2495.

[7] R. Espinosa-Luna, E. Bernabeu, On the $Q(M)$ depolarization metric, Opt. Commun. 277 (2007) 256-258.

[8] R. Espinosa-Luna, E. Bernabeu, G. Atondo-Rubio, $Q(M)$ and the depolarization index scalar metrics, Appl. Opt. 47 (2008) 1575-1580.

[9] J.J. Gil, Polarimetric characterization of light and media, Eur. Phys. J. Appl. Phys. 40 (2007) 1-47.

[10] R.M.A. Azzam, N.M. Bashara, Ellipsometry and Polarized Light, North-Holland, Amsterdam, 1989.

[11] M. Born, E. Wolf, Principles of Optics, Seventh (expanded) ed., Cambridge University Press, 2005.

[12] D. Goldstein, Polarized Light, Marcel Dekker, New York, 2003.

[13] C. Brosseau, Fundamentals of Polarized Light: A Statistical Optics Approach, Wiley, New York, 1998.

[14] G. Atondo-Rubio, R. Espinosa-Luna, A. MendozaSuárez, Mueller matrix determination for one-dimensional rough surfaces with a reduced number of measurements, Opt. Commun. 244 (2005) 7-13.

[15] O.G. Rodríguez-Herrera, N.C. Bruce, Mueller matrix for an ellipsoidal mirror, Opt. Eng. 45 (2006) 1-6 Art. 053602.

[16] R.A. Chipman, Polarimetry, in: Handbook of Optics, vol. II, McGraw Hill, New York, 1995 (Chapter 22). 
[17] M.W. Williams, Depolarization and cross polarization in ellipsometry of rough surfaces, Appl. Opt. 25 (1986) 3616-3622.

[18] J. Cariou, B. Le Jeune, J. Lotran, Y. Guern, Polarization effects of seawater and underwater targets, Appl. Opt. 29 (1990) 1689-1695.

[19] C. Collet, J. Zallat, Y. Takakura, Clustering of Mueller matrix images for skeletonized structure detection, Opt. Express 12 (2004) 1271-1280.

[20] S. Manhas, M.K. Swami, P. Buddhiwant, N. Ghosh, P.K. Gupta, K. Singh, Mueller matrix approach for determination of optical rotation in chiral turbid media in backscattering geometry, Opt. Express 14 (2006) 190-202.
[21] M.H. Smith, Optimization of a dual-rotating-retarder Mueller matrix polarimeter, Appl. Opt. 41 (2002) 2488-2493.

[22] B.J. Howell, Measurement of the polarization effects of an instrument using partially polarized light, Appl. Opt. 18 (1979) 809-812.

[23] A. Aiello, G. Puentes, D. Voigt, J.P. Woerdman, Maximum likelihood estimation of Mueller matrices, Opt. Lett. 31 (2006) 817-819.

[24] L. Giudicotti, M. Brombin, Data analysis for a rotating quarter-wave, far-infrared Stokes polarimeter, Appl. Opt. 46 (2007) 2638-2648. 